\title{
REVISTAMATĒRIA
}

\section{Materiais celulares vítreos obtidos via colagem de gel de uma emulsão de óleo vegetal}

\section{Cellular vitreous materials obtained by gel slip casting of a vegetable oil emulsion}

\author{
Francielly Roussenq Cesconeto ${ }^{1}$, Sabrina Arcaro ${ }^{1}$, \\ Bianca Goulart de Oliveira Maia ${ }^{1}$, Marcelo Tramontin Souza ${ }^{1}$, \\ João Batista Rodrigues Neto ${ }^{1}$, Antonio Pedro Novaes de Oliveira ${ }^{1}$
}

\footnotetext{
${ }^{1}$ Programa de Pós-Graduação em Ciência e Engenharia de Materiais (PGMAT), Laboratório de Materiais Vitrocerâmicos (VIROCER), Universidade Federal de Santa Catarina ( UFSC). Campus Universitário - Trindade, Caixa Postal 476, 88040-900 Florianópolis, SC.

e-mail: bianca.engenharia@gmail.com
}

\begin{abstract}
RESUMO
Resíduos de vidro podem ser reutilizados para o desenvolvimento de materiais alternativos. Uma opção viável são os materiais celulares para isolamentos térmicos já que, apresentando poros isolados, possuem baixa condutividade térmica, baixa densidade aparente e temperaturas de serviço mais altas que a dos materiais isolantes poliméricos. Neste trabalho, foi preparada uma suspensão aquosa com resíduos de vidro previamente moídos, Ágar-ágar (gelificante) e Emustab ${ }^{\circledR}$ (emulsificante), a qual foi adicionada diferentes frações de óleo de soja (50, 70 e 90\% em massa). Subsequentemente, foi realizada a emulsificação do óleo vegetal no líquido da suspensão por meio de agitação em misturador mecânico de alto cisalhamento. Corpos de prova cilíndricos foram produzidos via colagem por gelificação e queimados a $700{ }^{\circ} \mathrm{C} / 30 \mathrm{~min}$. Os resultados mostraram que os vidros celulares obtidos apresentaram porosidade entre 74 e $93 \%$ com poros bem definidos e distribuídos e baixas condutividades térmicas $(0,05$ a $0,09 \mathrm{~W} / \mathrm{m} . \mathrm{K})$. Tais resultados indicam que os materiais obtidos são potenciais candidatos para atuarem como isolantes térmicos, como por exemplo, painéis para revestimentos utilizados na construção civil.
\end{abstract}

Palavras-chave: Emulsões, vidros celulares, resíduos de vidro.

\begin{abstract}
Glass wastes can be reused for the development of alternative materials. A viable option are the cellular materials for thermal insulation since with isolated pores they show low thermal conductivity, low density and service temperatures higher than those of the polymeric insulating materials. In this work an aqueous suspension was prepared with previously milled glass wastes, agar (gelling) and Emustab ${ }^{\circledR}$ (emulsifier), in which different soy oil fractions (50, 70 and $90 \mathrm{wt} \%$ ) were added. Subsequently, the emulsification of the vegetable oil was carried out in the liquid suspension by stirring in a mechanical mixer at high shear. Cylindrical samples were produced by gelation slip casting and fired at $700{ }^{\circ} \mathrm{C} / 30 \mathrm{~min}$. The results showed that the obtained cellular glasses had porosity between 74 and 93\% with well-defined and distributed pores and low thermal conductivity $(0.05$ to $0.09 \mathrm{~W} / \mathrm{mK})$. These results indicate that the obtained materials are potential candidates to act as thermal insulators, such as panels for coatings used in construction.
\end{abstract}

Keywords: Emulsions, cellular glasses, glass wastes.

\section{INTRODUÇÃO}

O volume de vidro descartado no Brasil (garrafas, vasilhames diversos e vidros planos da construção civil) corresponde a 50\% do montante produzido anualmente, o que, de uma forma geral, representa milhares de toneladas de resíduos de vidros diariamente rejeitados e acumulados como lixo gerando, assim, um sério problema tanto do ponto de vista econômico quanto ambiental [1,2].

O vidro pode ser reciclado inúmeras vezes sem perder sua qualidade constituindo assim uma fonte de matéria-prima e de economia de energia. De fato, em geral, 55\% dos custos de produção estão relacionados à 
aquisição de matérias-primas, 30\% à mão de obra e 15\% ao consumo de energia [1]. Cada quilograma de vidro reciclado, na forma triturada, substitui 6,6 quilogramas de areia de quartzo e a refusão de 1 tonelada de vidro consome, em média, $70 \%$ menos energia do que a requerida para a fabricação de vidros a partir das matérias-primas naturais usuais [2,3]. Além do mais, vidros produzidos com material reciclado reduzem a quantidade de emissão de poluentes no ar em cerca de $20 \%$ e na água na ordem de $50 \%$ [3].

Uma opção para o reaproveitamento de resíduos de vidros é o desenvolvimento de produtos tecnológicos com maiores valores agregados que os dos produtos "in natura” [1,4], como por exemplo, vidros celulares. Espumas vítreas são materiais de elevada porosidade, não inflamáveis, resistentes química e mecanicamente e, possuem baixa condutividade térmica. Os vidros e assim as cerâmicas celulares encontram aplicações em diversas áreas de tecnologias, como na filtração de metais fundidos e gases quentes, queimadores radiantes porosos, componentes estruturais leves, catalisadores, sensores, reatores, bem como isolamentos acústicos e térmicos. Estes materiais são caracterizados por elevada porosidade ( $>60 \%$ vol.) com poros fechados e/ou abertos. A configuração e a quantidade de poros determinam propriedades específicas, tais como, baixa densidade e condutividade térmica, elevada área superficial, permeabilidade e estabilidade química e térmica $[5,6,7]$.

Conforme SILVEIRA et al. [8], diferentes processos podem ser utilizados para a obtenção desta classe de materiais como o método da réplica polimérica, geração de bolhas em uma suspensão, sinterização controlada, sol-gel, pirólise de aditivos orgânicos, entre outros. Devido a grande variedade de processos é possível obter microestruturas com diferentes tamanhos de células que podem variar em uma ampla faixa que vai de milímetros a nanômetros.

A obtenção de cerâmicas celulares por meio da gelificação consiste na emulsificação de um líquido apolar em água, por exemplo, óleos vegetais. Estes são extraídos de plantas e constituídos basicamente de triacilgliceróis (> 95\% massa), produtos resultantes da esterificação entre o glicerol e ácidos graxos [9]. Nesses sistemas, um líquido está disperso em outro na forma de gotículas, geralmente estabilizadas por um emulsificante, tal como o emustab, que se localiza na interface entre as fases líquidas, reduzindo a tensão interfacial [10]. Em uma etapa posterior, faz-se a adição de substâncias gelificantes, por exemplo, o àgar-àgar, para assim formar uma estrutura tridimensional que retém as moléculas de água nos seus interstícios, convertendo um fluido viscoso em um sólido elástico, que é posteriormente conformado, seco e submetido a um tratamento térmico. Por meio desta técnica é possível controlar, com precisão, a porosidade do material (que resulta da fração de líquido apolar adicionado à suspensão cerâmica) e o tamanho dos poros através do controle do tamanho das gotículas da fase emulsificada [10].

Levando em consideração o grande volume de vidros descartados, existe a oportunidade de novas aplicações para esse material. Neste trabalho, vidros celulares processados a partir de resíduos (vidros de garrafas) foram obtidos por meio de emulsificação de óleo vegetal de soja em suspensão aquosa seguida de gelificação, secagem e tratamento térmico para produção de isolantes térmicos porosos.

\section{MATERIAIS E MÉTODOS}

Para a realização deste trabalho foram utilizados os seguintes materiais: resíduos de vidro de garrafas transparentes como matriz cerâmica, óleo de soja como agente porogênico, emustab ${ }^{\circledR}$ como agente emulsificante e ágar-ágar como gelificante.

As garrafas de vidro transparentes foram trituradas em um britador de martelos (Servitech, CT-058) e os fragmentos foram moídos a seco por 90 min em moinho de jarro rápido de laboratório (Servitech, CT-242) com bolas de alumina. Na sequência, o pó obtido foi peneirado em malha 325 mesh $(45 \mu \mathrm{m})$. A análise química do pó de vidro foi feita por espectroscopia de fluorescência de raios X, FRX (Bruker, modelo S2 Ranger).

Uma amostra do vidro foi aquecida a $10^{\circ} \mathrm{C} / \mathrm{min}$ até a fusão (atmosfera oxidante) em um dilatômetro óptico (Expert System Solution, Misura ODHT) tal que uma curva de retração térmica linear foi obtida. A partir desta curva foi possível determinar o início e o fim do processo de densificação do pó de vidro de garrafa empregado neste trabalho.

Para determinar as temperaturas de início e fim de decomposição dos aditivos utilizados e a perda de massa associada aos mesmos, durante aquecimento a partir da temperatura ambiente $\left(\sim 25^{\circ} \mathrm{C}\right)$, foram realizadas análises termogravimétricas (ATG) em um equipamento TA Instruments, SDT Q600, a $10{ }^{\circ} \mathrm{C} / \mathrm{min}$ em atmosfera oxidante até $1200{ }^{\circ} \mathrm{C}$. Neste caso, amostras de $30 \mathrm{mg}$ foram pesadas e colocadas em cadinho de platina e um mesmo cadinho de platina vazio foi utilizado como material de referencia.

Para obtenção das espumas, foi preparada uma solução aquosa de ágar-ágar a $90{ }^{\circ} \mathrm{C}(20 \%$ em massa em relação à água) a qual, posteriormente, foi adicionado pó de vidro (60\% vol. de sólidos) e emustabß (20\% em massa em relação à fração de óleo). A suspensão foi desaglomerada em uma batedeira doméstica (Philips Walita) por 3 min. Durante a agitação, o óleo (50, 70 e 90\% em massa em relação à suspensão) foi lentamente adicionado até a completa emulsificação. Na sequência, esta emulsão cerâmica foi rapidamente 
vazada em moldes cilíndricos (com $30 \mathrm{~mm}$ de diâmetro e $25 \mathrm{~mm}$ de altura - dimensões nominais) e os corpos de prova foram secos a temperatura ambiente $\left(\sim 25^{\circ} \mathrm{C}\right)$ por cinco dias.

A queima das amostras produzidas foi realizada em forno tipo mufla (Jung, J200) com aquecimento a uma taxa de $0,5^{\circ} \mathrm{C} / \mathrm{min}$ até $600^{\circ} \mathrm{C}$ com um patamar de 30 minutos (para eliminação dos componentes orgânicos) seguindo a $10^{\circ} \mathrm{C} / \mathrm{min}$ até $700^{\circ} \mathrm{C}$ com patamar de 30 minutos, para sinterização.

A densidade aparente das espumas foi determinada relacionando as suas medidas geométricas, obtidas por meio de um paquímetro (Mitutoyo, precisão de $\pm 0,01 \mathrm{~mm}$ ), às suas massas, obtidas por meio de balança analítica (Shimadzu, AX200, precisão de 0,001 g). As densidades relativas $\left(\rho_{\mathrm{r}}\right)$ foram determinadas relacionando-se (razão) as densidades aparentes $\left(\rho_{\mathrm{a}}\right)$ e a densidade verdadeira $\left(\rho_{\mathrm{v}}\right)$ do pó de vidro moído, determinada por picnometria gasosa (Quantachrome Ultrapycnometer, 1000 (LIMAC)). Assim e por meio da equação 1 , as porosidades $(\varepsilon)$ foram calculadas.

$$
\varepsilon=\left(1-\rho_{\mathrm{r}}\right) \times 100
$$

A microestrutura de poros pôde ser visualizada a partir de imagens das superfícies de fratura das amostras queimadas, obtidas em microscópio óptico, MO (OPTON IS130). A condutividade térmica das espumas foi determinada em um equipamento TCi Thermal Conductivity Analayzer (C-THERM TECHNOLOGIES).

\section{RESULTADOS E DISCUSSÃO}

A Tabela 1 apresenta o resultado da análise química referente ao resíduo de vidro.

Tabela 1: Composição química do vidro de garrafa reciclado.

\begin{tabular}{cccccccccc}
\hline \multicolumn{10}{c}{ ÓXIDOS CONSTITUINTES (\% MASSA) } \\
\hline $\mathrm{SiO}_{2}$ & $\mathrm{Na}_{2} \mathrm{O}$ & $\mathrm{CaO}$ & $\mathrm{Al}_{2} \mathrm{O}_{3}$ & $\mathrm{Fe}_{2} \mathrm{O}_{3}$ & $\mathrm{~K}_{2} \mathrm{O}$ & $\mathrm{P}_{2} \mathrm{O}_{5}$ & $\mathrm{TiO}_{2}$ & P.F. \\
70,60 & 16,68 & 9,56 & 2,15 & 0,10 & 0,02 & 0,02 & 0,04 & 0,83 \\
\hline
\end{tabular}

P.F.: Perda ao Fogo.

Os principais óxidos constituintes do vidro são o $\mathrm{SiO}_{2}$ (formador da fase vítrea), o $\mathrm{Na}_{2} \mathrm{O}$ (utilizado para reduzir a temperatura de processamento) e o $\mathrm{CaO}$ (torna o vidro insolúvel e proporciona estabilidade contra agentes atmosféricos). Pode-se observar também uma pequena quantidade de $\mathrm{Al}_{2} \mathrm{O}_{3}$ utilizada para evitar a devitrificação (cristalização) do vidro e melhorar a sua resistência mecânica e ao choque térmico.

Para definição do tratamento térmico a ser empregado na consolidação dos materiais celulares produzidos com o resíduo de vidro, fez-se necessário entender o comportamento deste submetido a um aquecimento controlado. A Figura 1 apresenta uma curva de retração linear (RL) do resíduo de vidro em função da temperatura.

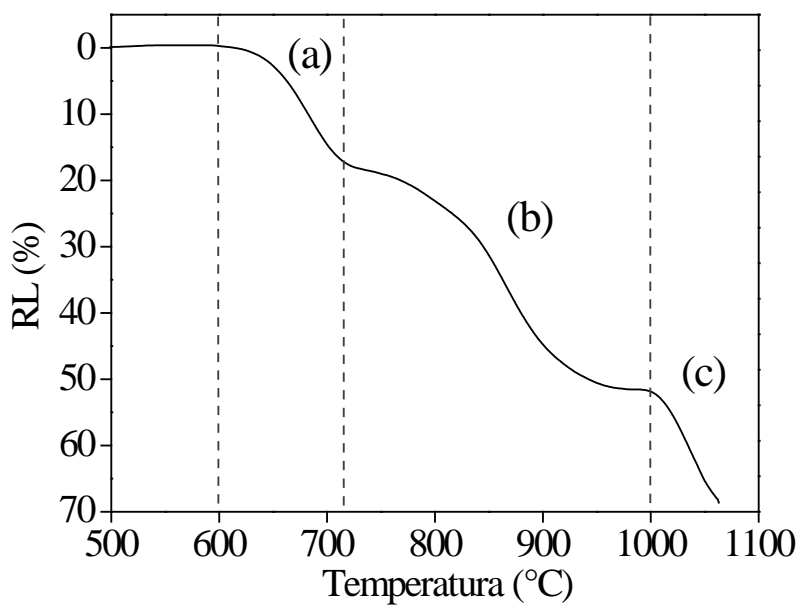

Figura 1: Retração linear (RL) do resíduo de vidro.

Observam-se, na Figura 1, três fenômenos em termos de retração linear: (a) o início da retração/densificação do compactado de vidro em torno de $600{ }^{\circ} \mathrm{C}$ com máximo em torno de $720{ }^{\circ} \mathrm{C}$ o qual cor- 
responde a 20\% de retração linear; o segundo fenômeno (b) refere-se ao amolecimento do vidro, o qual tem início em torno de $720^{\circ} \mathrm{C}$; e, por último, (c) a fusão (temperatura de esfera) do vidro, a partir de $1000^{\circ} \mathrm{C}$.

Com a finalidade de determinar a temperatura de eliminação dos compostos orgânicos que estruturam o vidro celular a verde, foram realizadas análises de calorimetria diferencial exploratória (DSC) e termogravimétricas (TG), apresentadas na Figura 2, do (a) emustab ${ }^{\circledR}$, (b) ágar-ágar, (c) óleo de soja e (d) uma espuma vítrea produzida com 70\% em massa de óleo de soja.

A perda de massa do emustab ${ }^{\circledR}$ (Figura 2(a)), desde a temperatura ambiente (início do ensaio) até cerca de $500{ }^{\circ} \mathrm{C}$ dá-se, basicamente, pela eliminação de água e dos compostos orgânicos de natureza lipídica (monoglicerídeos destilados, monoestearato de sorbitana e polissorbato 60) que constituem o emulsificante [11]. O ágar-ágar (Figura 2(b)) também inicia sua decomposição progressiva até cerca de $570{ }^{\circ} \mathrm{C}$ a qual está relacionada à vaporização da água e de polissacarídeos (agarose e agaropectina).
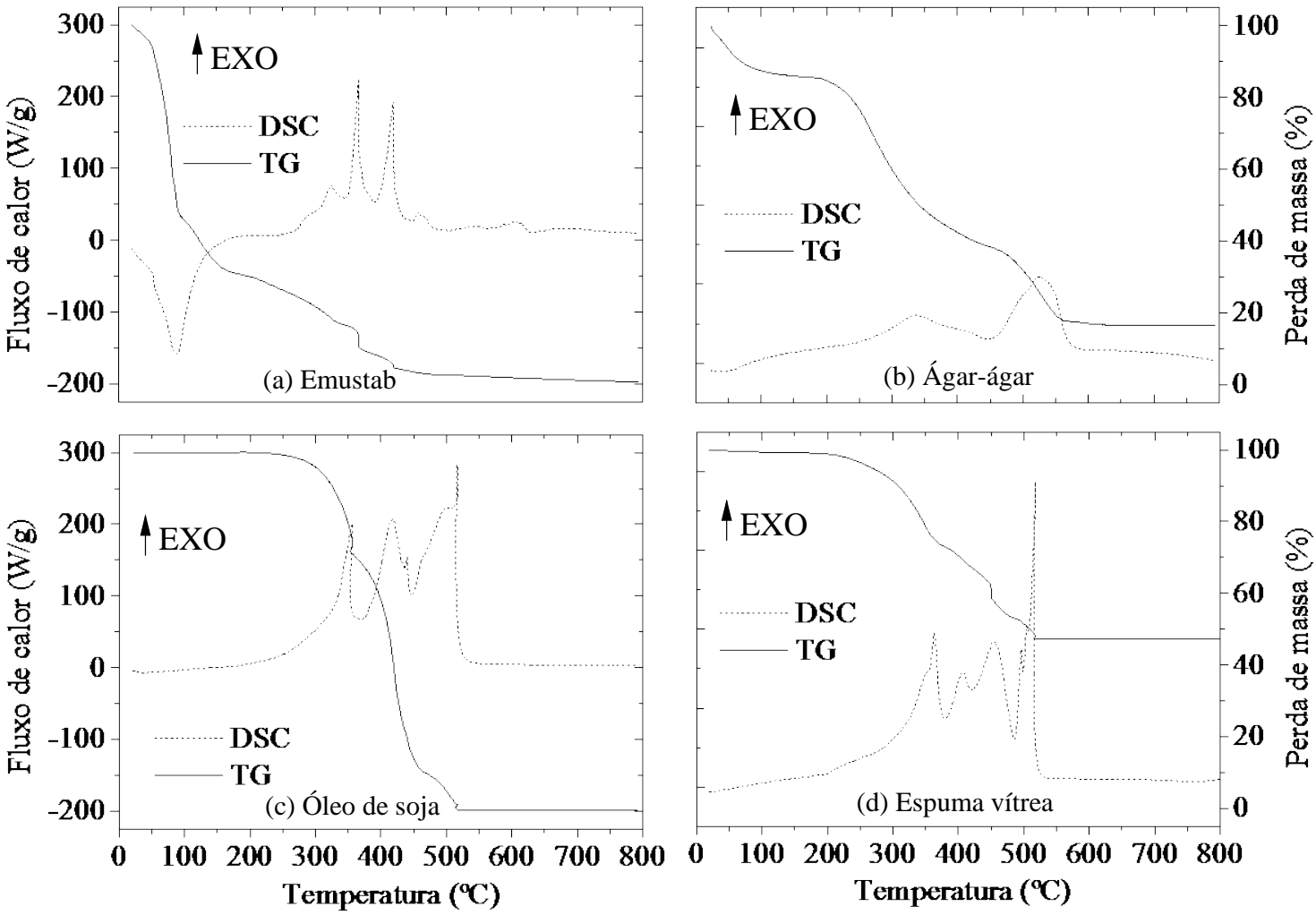

Figura 2: Análises de calorimetria diferencial exploratória (DSC) e termogravimétricas (TG) dos materiais: (a) emustab®, (b) ágar-ágar, (c) óleo de soja e (d) uma espuma vítrea produzida com 70\% em massa de óleo de soja.

Na Figura 2(c), observa-se que a perda de massa do óleo de soja ocorre entre 220 e $520{ }^{\circ} \mathrm{C}$ e, corresponde basicamente à eliminação de triacilgliceróis provenientes do óleo vegetal [9]. A Figura 2(d) mostra o comportamento da espuma vítrea que corrobora com os resultados dos reagentes apresentados nas Figuras 2 (a), (b) e (c).

Após, as análises térmicas do vidro e do vidro celular verde e conhecendo-se a região de temperatura que ocorre a densificação do vidro $\left(600-700^{\circ} \mathrm{C}\right)$ e a eliminação dos orgânicos que estruturam os poros do vidro celular (até $500^{\circ} \mathrm{C}$ ), foi possível definir um ciclo térmico que permitisse, ao mesmo tempo, a eliminação dos componentes orgânicos e a densificação da estrutura vítrea (arestas) sem que ocorresse o colapso das amostras (corpos pré-formados). Assim, o ciclo térmico definido para queima compreendeu os seguintes estágios: aquecimento a $0,5{ }^{\circ} \mathrm{C} / \mathrm{min}$ até $600{ }^{\circ} \mathrm{C}$ com um patamar de 30 minutos, seguindo a $10{ }^{\circ} \mathrm{C} / \mathrm{min}$ até $700{ }^{\circ} \mathrm{C}$ por 30 minutos.

A Figura 3 mostra os valores de condutividade térmica $(\mathrm{k})$ e porosidade $(\varepsilon)$ obtidos para os vidros celulares preparados com diferentes frações de óleo (50, 70 e 90\% em massa) após processo de queima. Os materiais preparados com 50, 70 e 90\% de óleo, apresentaram porosidades de 74, 85 e 93\%, respectivamente. 


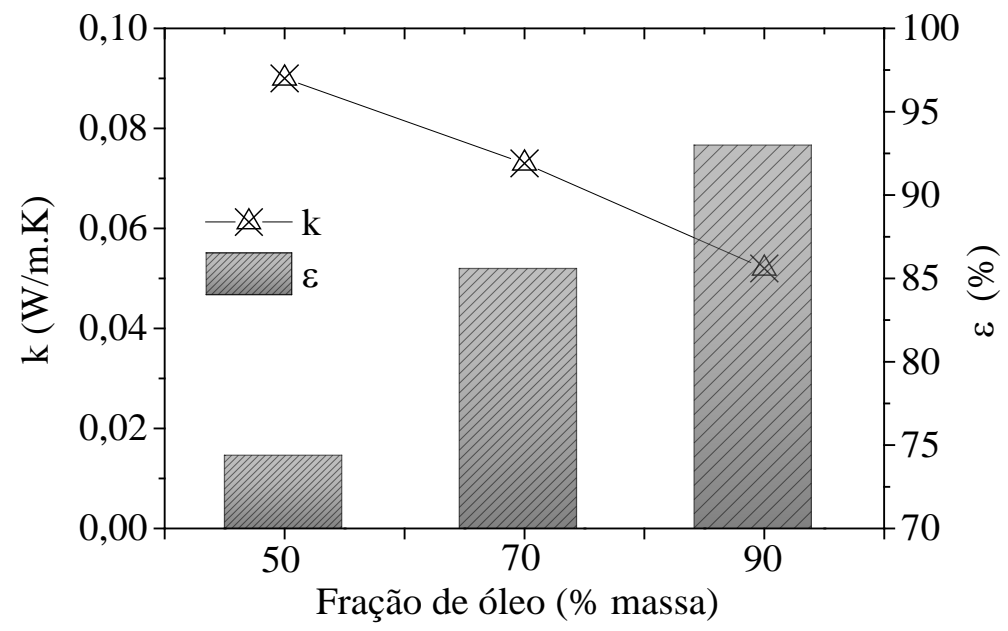

Figura 3: Condutividade térmica $(\mathrm{k})$ e porosidade $(\varepsilon)$ dos vidros celulares após processo de queima $\left(0,5^{\circ} \mathrm{C} / \mathrm{min}\right.$ até 600 ${ }^{\circ} \mathrm{C}$ com um patamar de $30 \mathrm{~min}$ e a $10{ }^{\circ} \mathrm{C} / \mathrm{min}$ até $700{ }^{\circ} \mathrm{C}$ por $30 \mathrm{~min}$ em função da concentração do agente porogênico) para frações crescentes de óleo de soja.

Pode-se perceber, como esperado, um aumento da porosidade com o aumento da fração de óleo de soja (agente porogênico). Observa-se ainda, na Figura 3, que os valores de condutividade térmica diminuem com o aumento da fração mássica de óleo de soja, em contraste com o aumento da porosidade ( $\varepsilon$ ), com valores entre 0,05 e $0,09 \mathrm{~W} / \mathrm{m} . \mathrm{K}$.

Materiais celulares vítreos têm sua temperatura de trabalho limitada a sua temperatura de transição vítrea (normalmente entre 530 e $550{ }^{\circ} \mathrm{C}$ ) ou até a sua temperatura de amolecimento (dependendo do caso), cujo fenômeno está associado a uma expansão abrupta [12]. Assim, a transferência de calor nesses materiais é governada basicamente pela condução, dado que nessa faixa de temperatura a radiação pode ser desprezada [13] e, de acordo com COLLISHAW e EVANS (1994), em espumas com tamanhos de poros inferiores a 4 mm, a transferência de calor por convecção também pode ser desprezada [14].

A Figura 4 mostra imagens dos vidros celulares preparados com diferentes frações de óleo de soja (a) 50, (b) 70 e (c) $90 \%$ após queima.
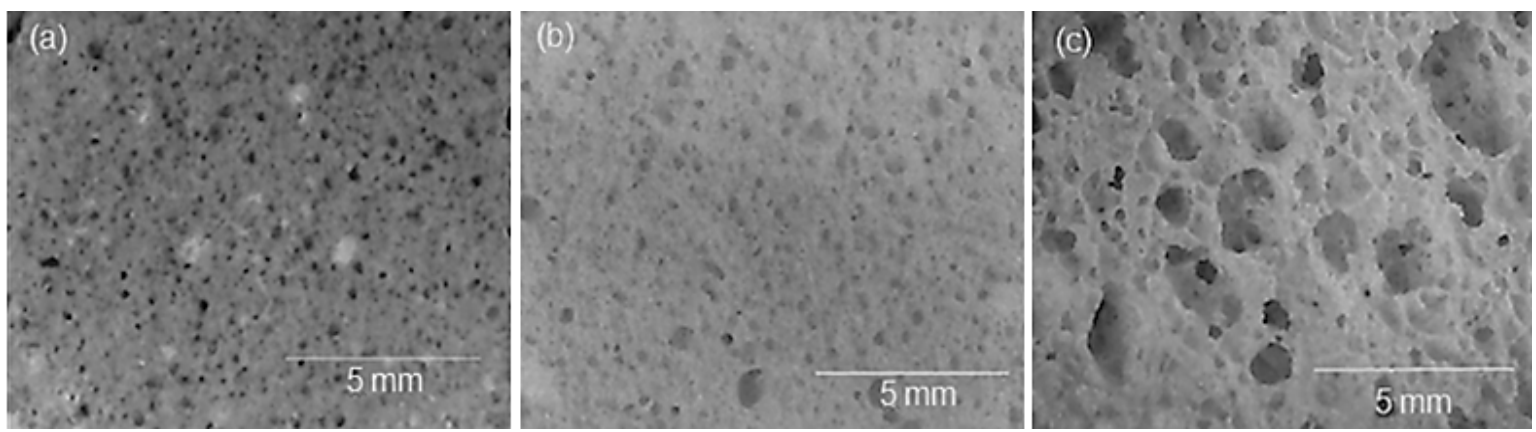

Figura 4: Imagens (MO) de fraturas dos vidros celulares, após queima $\left(0,5{ }^{\circ} \mathrm{C} / \mathrm{min}\right.$ até $600{ }^{\circ} \mathrm{C}$ com um patamar de 30 min e a $10{ }^{\circ} \mathrm{C} / \mathrm{min}$ até $700{ }^{\circ} \mathrm{C}$ por $30 \mathrm{~min}$ ) preparados com diferentes frações de óleo de soja (a) 50\%, (b) $70 \%$ e (c) $90 \%$ em massa.

Observa-se claramente que, para maiores proporções do agente porogênico, os poros tendem a aumentar de tamanho, porém com dimensões não superiores a 4 mm (Figura 4 (c)). Nota-se ainda que, na Figura 4, para a maior concentração de óleo (90\%), os poros tendem a uma estrutura aberta, dado que a formação excessiva de poros promove a coalescência e posterior cremeação das bolhas gasosas. Além disso, percebe-se que, em todas as amostras, a distribuição de tamanho de poros é homogênea.

De acordo com SCHEFFLER e COLOMBO (2005) espumas vítreas comerciais apresentam, tipicamente, valores de condutividade térmica na faixa de 0,04 a $0,08 \mathrm{~W} / \mathrm{m} . \mathrm{K}$ e porosidades entre 85 e $95 \%$ [7]. LI et al., (2013) produziram cerâmicas celulares pelo processo de gel-casting e obtiveram materiais com $81,1 \%$ de porosidade e condutividade térmica da ordem de 0,055 W/m.K [15]. 
Portanto, de acordo com os valores obtidos de porosidade e condutividade térmica, as espumas celulares vítreas produzidas neste trabalho apresentam potencial para serem aplicadas como isolantes térmicos, visto que os resultados estão em conformidade com aqueles tipicamente encontrados para materiais com estas características.

\section{CONCLUSÕES}

Vidros celulares de resíduos de vidro de garrafas, sódico-cálcicos, foram obtidos por meio de emulsificação de óleo de soja (50, 70 e $90 \%$ em massa) em suspensão aquosa (60\% vol.), seguida de gelificação, secagem e tratamento térmico/queima $\left(0,5{ }^{\circ} \mathrm{C} / \mathrm{min}\right.$ até $600{ }^{\circ} \mathrm{C}$ com um patamar de 30 min e a $10{ }^{\circ} \mathrm{C} / \mathrm{min}$ até $700{ }^{\circ} \mathrm{C}$ por $30 \mathrm{~min}$ ). O processo possibilitou a obtenção de vidros celulares com porosidade compreendida entre 74 e 93\% com poros bem definidos e distribuídos. Os valores de condutividade térmica diminuem com o aumento do teor do agente porogênico, isto é, de 0,90 a 0,52 W/m.K para 50 a $90 \%$ em massa de óleo, respectivamente, e são característicos de vidros celulares empregados como isolantes térmicos. Portanto, as propriedades obtidas mostram que essa rota de processamento é adequada quando o objetivo é obter materiais com estruturas homogêneas e com elevadas porosidades e baixas condutividades térmicas.

\section{AGRADECIMENTOS}

Os autores agradecem a FAPESC/CNPq (PRONEX T.O. No 17431/2011-9).

\section{BIBLIOGRAFIA}

[1] ASSIS, O.B.G. “O uso de vidro reciclado na confecção de membranas para microfiltração”, Cerâmica, v.52, pp. 105-113, 2006.

[2] ABIVIDROS - Associação Técnica Brasileira das Indústrias Automáticas de Vidro, http://www.abividro.org.br/reciclagem-abividro/beneficios-da-reciclagem-do-vidro. Acessado em março de 2014.

[3] CEMPRE - Compromisso Empresarial para a Reciclagem, http://www.cempre.org.br. Acessado em março de 2014.

[4] LIMA, N.M.O., VELOSO, I.T.B.M., MORAIS, C.R.S., et al., "Reciclagem de embalagens de resíduos vítreos para confecção de artefatos de decoração”, In : III Conferência Internacional de Gestão de Resíduos Sólidos, São Paulo, SP, 2013.

[5] ZESCHKY, J., NEUBAUER, J., JASON LO, S.H., et al. "Preceramic polymer derived cellular ceramics", Compos. Sci. Technol. v.63, pp. 2361-2370, 2003.

[6] BARG, S., SOLTMANN, C., ANDRADE, M., et al. "Cellular Ceramics by Direct Foaming of Emulsified Ceramic Powder Suspensions”, J. Am. Ceram. Soc. v.91, pp. 2823 - 2829, 2008.

[7] SCHEFFLER, M., COLOMBO, P., Cellular Ceramics: Structure, Manufacturing, Properties and Applications, Wiley VCH, Germany 2005.

[8] SILVEIRA, C.B., ESCOBAR, J.A., QUINTERO, M.W., et al. "Decomposição térmica de espumas de poliuretano para fabricação de vitrocerâmica celular de $\mathrm{Li}_{2} \mathrm{O}-\mathrm{ZrO}_{2}-\mathrm{SiO}_{2}-\mathrm{Al}_{2} \mathrm{O}_{3}$ (LZSA)”, Química Nova, v. 30, n. 5, pp.1104-1107, 2007.

[9] FONSECA, M.M., YOSHIDA, M.I. “Análise térmica do óleo de linhaça natural e oxidado”, Vértices, v.11, n.1/3, pp. 61-75, 2009.

[10] SOUSA, E., DELLÚ JR. M., PANDOLFELLI, V.C., et al. "Produção de cerâmicas celulares por emulsão seguida de gelificação”, Cerâmica, v. 57, pp. 38-44, 2011.

[11] BREDA, C.A., JUSTI, P.N., SANJINEZ-ARGANDOÑA, E.J. "Efeito da desidratação foam mat na retenção da vitamina C da polpa de cajá-manga”, Braz. J. Food Nutr., v.24, n.2, pp. 189-193, 2013.

[12] VARSHNEYA, A.K. Fundamentals of Inorganic Glasses, Society of Glass Technology, $2^{\mathrm{a}}$ ed., Sheffield, 2006.

[13] DEWITT, P.D., INCROPERA, P.F., BERGMAN, T.L., et al. Fundamentos da Transferência de calor e de massa, $7^{\mathrm{a}}$ ed. LTC, 2014.

[14] COLLISHAW, P.G., EVANS, J.R.G. “An assessment of expressions for the apparent thermal conductivity of cellular materials”, Journal of materials science, v.29, pp. 486-498, 1994.

[15] LI, S., WANG, C. A., ZHOU J. "Effect of starch addition on microstructure and properties of highly porous alumina ceramics”, Ceramics International, v.39, n.8, pp. 8833-8839, 2013. 